\title{
Ensayo de aptitud realizado en LATU para validación del método de calibración y uso de pipetas de vidrio y pipetas automáticas
}

\author{
Santo, C. ${ }^{(1)}$, Constantino, P. ${ }^{(1)}$, Sica, A. ${ }^{(1)}$ \\ Contacto: csanto@latu.org.uy \\ (1) Departamento de Metrología, Laboratorio Tecnológico del Uruguay (LATU) \\ Recibido: 11/9/2008 - Aprobado: 2/12/2008
}

\begin{abstract}
$\underline{\text { Resumen }}$
En 2008 se realizó un ensayo de aptitud para calibración de material volumétrico, con la participación de varios analistas (en su mayoría nuevos en la tarea) de los laboratorios de ensayo del Laboratorio Tecnológico del Uruguay (LATU). Para el mismo se utilizaron dos pipetas, una de vidrio de $1 \mathrm{ml}$ y una pipeta automática de volumen regulable entre 0 y $1 \mathrm{ml}$, ésta última calibrada para el valor correspondiente a $1 \mathrm{ml}$. Con el fin de evaluar la repetibilidad en la medición, cada analista repitió entre 6 y 10 veces la calibración con cada pipeta. Se evaluó la media aritmética de las mediciones de cada analista para cada pipeta y se estimó su incertidumbre expandida. Aquí se presentan los gráficos con las medias y las incertidumbres obtenidas para cada tipo de pipeta y se efectúa un estudio comparativo de los resultados. A modo de conclusión se evaluó la reproducibilidad de cada tipo de instrumento, en base a lo cual se formulan recomendaciones para su uso y calibración.

Palabras clave: material volumétrico, pipeta, ensayo de aptitud, calibración.
\end{abstract}

\begin{abstract}
In 2008 several chemical analysts from LATU's test laboratories (most of them new to their task) took part in a Proficiency Test for the use of volumetric glassware. Two pipettes were used: a glass pipette of $1 \mathrm{ml}$, class B, and an automatic pipette of variable volume between $0 \mathrm{ml}$ and $1 \mathrm{ml}$. The latter was calibrated at $1 \mathrm{ml}$. All analysts were asked to repeat each calibration between 6 and 10 times to allow an estimate of the measurement repeatibility. The average value performed by each analyst for each pipette was evaluated and its uncertainty estimated. Graphs are presented showing the analysts' means and uncertainties for each pipette and the results are compared. As a conclusion, the reproducibility of each kind of instrument is evaluated according to which recommendations are given for the calibration and use of the two types of pipette.

Key words: glassware, pipette, Proficiency Test, calibration.
\end{abstract}

\section{Introducción}

El LATU es una institución certificada por la norma ISO 9001 y aproximadamente 250 de los ensayos químicos y físicos que se realizan se encuentran acreditados por la norma ISO 17025. Está implementado por lo tanto un Sistema de Gestión que asegura la calidad de los resultados emitidos. En el punto 7.6 de la ISO 9001, así como en el punto 5.6 de la ISO 17025, se establece la necesidad de utilizar equipamiento calibrado durante la realización de los ensayos.

Dada la dimensión de la institución, que integra diferentes departamentos analíticos especializados, la cantidad de material volumétrico que se utiliza es muy grande. En el pasado todo el material volumétrico era calibrado en el Departamento de Metrología Científica. Para mejorar la gestión de los recursos y la calidad de los resultados analíticos, se implementó la política de calibrar el material volumétrico por parte del personal de cada uno de los departamentos que hacen uso del mismo. Esta política presenta la ventaja de asegurar que el material se calibre en las mismas condiciones y con la misma metodología con que se usa, disminuyendo de esta forma los errores en el uso y favoreciendo el entrenamiento del personal que utiliza el material volumétrico, haciéndolo participar del proceso de calibración.

Para evaluar la competencia del personal, la calibración del equipamiento y el aseguramiento de calidad en las mediciones en los distintos departamentos, se realizan ensayos de aptitud periódicos en calibración de material volumétrico en los que participan los nuevos analistas (de forma de validar la forma de uso del material volumétrico) y analistas más experimentados (para evaluar si sus resultados siguen dentro del rango de tolerancia).

Se debe tener en consideración que los problemas detectados en la calibración del material volumétrico reflejan problemas en el uso del mismo.

Frente a la gran cantidad de métodos químicos existentes que involucran, a su vez, un número creciente de matrices y analitos, se evidencia la falta de oferta de interlaboratorios y/o de materiales de referencia para cada método y en cada analito y matriz. En estos casos se debe recurrir al aseguramiento de calidad de los métodos químicos, asegurando metrológicamente cada subproceso involucrado (por ejemplo: uso de material volumétrico, extracciones, separación cromatográfica, etc.). En ese sentido resulta importante mantener en control el subproceso de uso de material volumétrico.

En cada ensayo de aptitud se seleccionan dos ítems de material volumétrico que son calibrados por técnicos del Departamento de Metrología para asignarles el valor de referencia. Los ítems se hacen circular según un plan especificado entre los analistas de los distintos departamentos analíticos del LATU para que procedan a su calibración utilizando igual procedimiento. Los resultados de calibración acompañados de sus respectivas incertidumbres son informados al organizador, que procesa los datos, los evalúa y elabora un reporte. Luego se realiza una reunión de devolución con los participantes, donde se discuten los resultados, se asignan causas probables a las tendencias y desviaciones y se evalúan posibles acciones correctivas y preventivas a implementar. 


\section{Materiales y Métodos}

En el caso del ensayo de aptitud, objeto del presente estudio, se evaluó la competencia de distintos analistas en el uso y calibración de una pipeta aforada de vidrio de $1 \mathrm{ml}$ y una pipeta automática de volumen variable calibrada en un volumen de $1 \mathrm{ml}$.

El método utilizado para la calibración es un procedimiento interno del LATU basado en las normas ISO 4787 e ISO 648.

Los resultados obtenidos sirvieron no solo para evaluar la calidad de las mediciones de volumen en los departamentos, sino también para comparar el comportamiento en el uso de estos dos tipos de pipetas, las fuentes de incertidumbre asociadas a cada una y su cuantificación.

Participaron 10 analistas de distintos departamentos del LATU, ocho de ellos pertenecientes al área química y los dos restantes al área de volumen del Departamento de Metrología.

El valor de referencia fue calculado como el promedio ponderado de los valores obtenidos por los técnicos del área de volumen del Departamento de Metrología (identificados como analistas 1 y 2), en el caso de la pipeta aforada, y por la media aritmética de los participantes en el caso de la pipeta automática. Esto se debe a que en el caso de material de vidrio aforado está implementado un sistema de aseguramiento de calidad en las mediciones por parte del Departamento de Metrología Científica que asegura resultados confiables en el marco de las normas ISO de referencia citadas. No es así para el caso de la pipeta automática, donde no existen normativas de uso, salvo las del fabricante. Los resultados de las mediciones de volumen son trazables al Sistema Internacional, ya que las capacidades de masa y temperatura están declaradas en el apéndice $\mathrm{C}$ del acuerdo de reconocimiento mutuo del CIPM (Comité Internacional de Pesas y Medidas).

\section{Resultados}

\section{Definiciones:}

$E_{n}=\frac{E}{\sqrt{\left(U^{2}{ }_{L A T U}+U^{2}{ }_{L A B}\right)}}$

$E_{n}$ - Error normalizado

$E-$ Error $=$ Valor informado por el analista - Valor de referencia

$U_{L A T U}$ - Incertidumbre asumida para el valor de referencia

NOTA: Se asumió para el valor de referencia una incertidumbre igual a la tolerancia definida para la pipeta, de forma de evaluar qué laboratorios se alejaban del valor de referencia más que el valor de la tolerancia, teniendo en cuenta la incertidumbre de la medición.

En el caso de la pipeta aforada se asumió un valor de tolerancia igual al correspondiente a una pipeta clase $\mathrm{B}$ y en el caso de la automática a un $0,5 \%$ del valor, según lo indica el fabricante.

$U_{L A B}$ - Incertidumbre en el valor informada por el analista.

Un error normalizado superior a 1 implica que el error en la medición es significativo, ya que relaciona por su definición el valor del error en la medición con la incertidumbre de dicho error.

Para evaluar el efecto de estos errores en el uso rutinario de una pipeta, si se sigue el mismo procedimiento que el utilizado en la calibración, el error relativo que se obtendría en un posible resultado, por ejemplo de una solución valorada preparada por cada analista participante, sería mayor o igual que el error relativo calculado a partir de los datos de error provenientes de las Tablas 1 o 2 .

\section{Resultados pipeta de vidrio}

\begin{tabular}{|c|c|c|c|c|c|c|c|c|c|c|}
\hline Analista & $\mathbf{1}$ & $\mathbf{2}$ & $\mathbf{3}$ & $\mathbf{4}$ & $\mathbf{5}$ & $\mathbf{6}$ & $\mathbf{7}$ & $\mathbf{8}$ & $\mathbf{9}$ & $\mathbf{1 0}$ \\
\hline $\begin{array}{c}\text { Resultado } \\
/ \mathrm{cm}^{3}\end{array}$ & 0,9612 & 0,9655 & 0,9696 & 0,9983 & 0,9677 & 0,9925 & 0,9995 & 0,9708 & 0,9800 & 0,9926 \\
\hline$U_{L A B} / \mathrm{cm}^{3}$ & 0,0077 & 0,0018 & 0,0044 & 0,0054 & 0,0073 & 0,0079 & 0,0029 & 0,0012 & 0,021 & 0,0084 \\
\hline $\begin{array}{c}\text { Valor de referencia/ } \\
\mathrm{cm}^{3}\end{array}$ & 0,9653 & 0,9653 & 0,9653 & 0,9653 & 0,9653 & 0,9653 & 0,9653 & 0,9653 & 0,9653 & 0,9653 \\
\hline$U_{L A T U} / \mathrm{cm}^{3}$ & 0,0150 & 0,0150 & 0,0150 & 0,0150 & 0,0150 & 0,0150 & 0,0150 & 0,0150 & 0,0150 & 0,0150 \\
\hline $\mathrm{E}_{\mathrm{cm}} \mathrm{cm}^{3}$ & $-0,0041$ & 0,0002 & 0,0044 & 0,0330 & 0,0024 & 0,0272 & 0,0342 & 0,0055 & 0,0147 & 0,0273 \\
\hline$E_{n}$ & $-0,24$ & 0,01 & 0,28 & 2,07 & 0,14 & 1,61 & 2,24 & 0,37 & 0,57 & 1,59 \\
\hline
\end{tabular}

Tabla 1. Resultados obtenidos para la pipeta aforada.

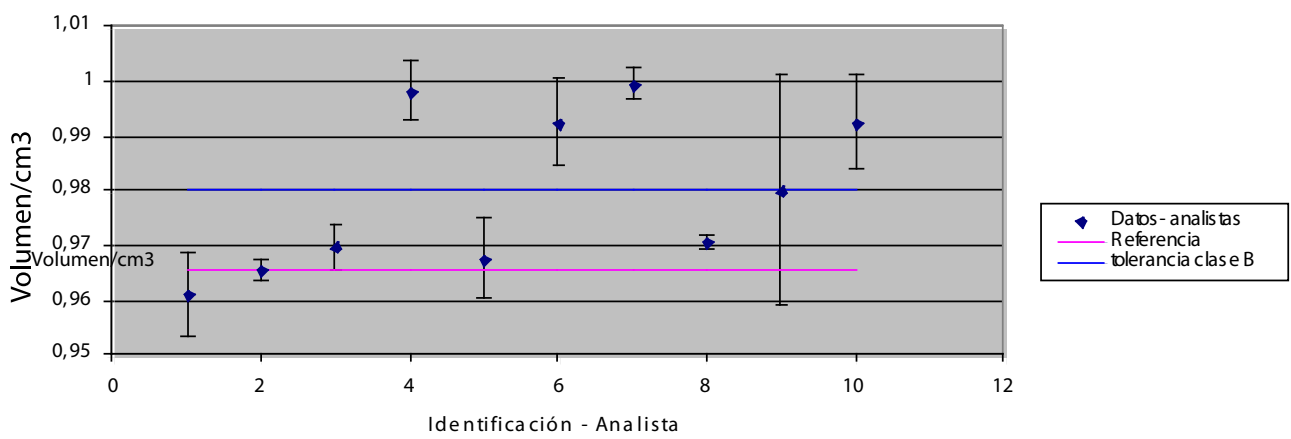

Figura 1. Gráfico de resultados con su incertidumbre- pipeta aforada 


\section{Resultados de pipeta automática}

\begin{tabular}{|c|c|c|c|c|c|c|c|c|c|c|}
\hline Laboratorio & 1 & 2 & 3 & 4 & 5 & 6 & 7 & 8 & 9 & 10 \\
\hline Resultado $/ \mathrm{cm}^{3}$ & 1,0035 & 1,0056 & 0,9933 & 1,0004 & 1 & 0,9993 & 0,9986 & 1,0028 & 0,9995 & 1,0053 \\
\hline$U_{L A B} / \mathrm{cm}^{3}$ & 0,0008 & 0,0008 & 0,0049 & 0,0044 & 0,0002 & 0,0007 & 0,0015 & 0,0008 & 0,0013 & 0,0032 \\
\hline $\begin{array}{c}\text { Valor de } \\
\text { referencia } / \mathrm{cm}^{3}\end{array}$ & 1,00083 & 1,00083 & 1,00083 & 1,00083 & 1,00083 & 1,00083 & 1,00083 & 1,00083 & 1,00083 & 1,00083 \\
\hline$U_{L A T U} / \mathrm{cm}^{3}$ & 0,005 & 0,005 & 0,005 & 0,005 & 0,005 & 0,005 & 0,005 & 0,005 & 0,005 & 0,005 \\
\hline$E / \mathrm{cm}^{3}$ & 0,0027 & 0,0048 & $-0,0075$ & $-0,0004$ & $-0,0008$ & $-0,0015$ & $-0,0022$ & 0,0020 & $-0,0013$ & 0,0045 \\
\hline$E_{n}$ & 0,53 & 0,94 & $-1,08$ & $-0,06$ & $-0,17$ & $-0,30$ & $-0,43$ & 0,39 & $-0,26$ & 0,75 \\
\hline
\end{tabular}

Tabla 2. Resultados obtenidos para la pipeta automática.

PIPETA AUTOMÁTICA

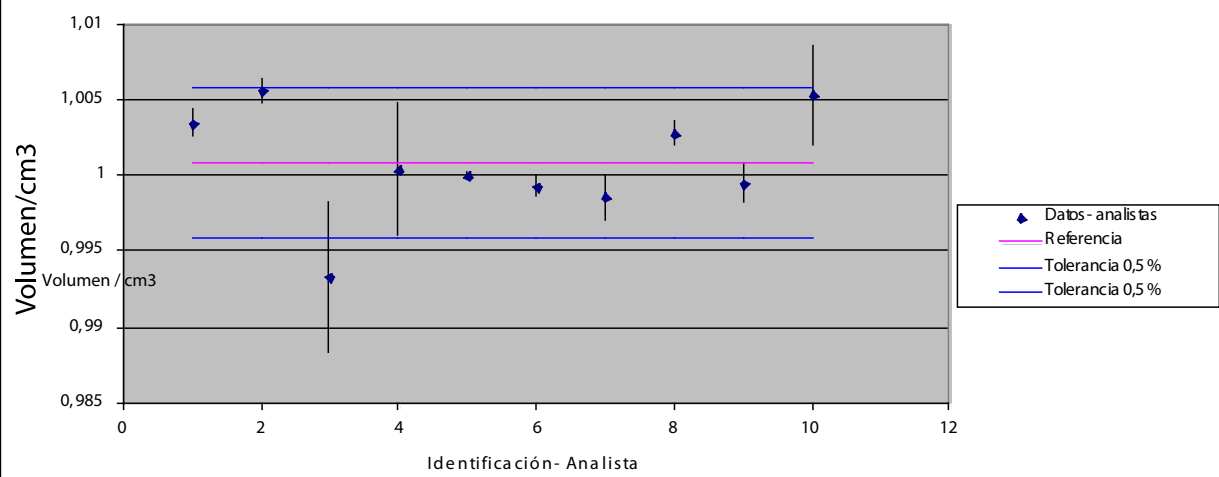

Figura 2. Gráfico de resultados con su incertidumbre- pipeta automática.

\section{Resultados de repetibilidad de las dos pipetas}

El valor de repetibilidad corresponde al desvío estándar obtenido a partir de los resultados de las repeticiones realizadas por cada analista para cada pipeta.

\begin{tabular}{|c|c|c|c|c|c|c|c|c|c|c|}
\hline ANALISTA & 1 & 2 & 3 & 4 & 5 & 6 & 7 & 8 & 9 & 10 \\
\hline PIPETA DE VIDRIO/ml & 0,0108 & 0,0024 & 0,0130 & 0,0024 & 0,0065 & 0,0110 & 0,0027 & 0,0019 & 0,0182 & 0,0030 \\
\hline PIPETA AUTOMÁTICA/ml & 0,0007 & 0,0009 & 0,0059 & 0,0050 & 0,0027 & 0,0009 & 0,0019 & 0,0009 & 0,0017 & 0,0017 \\
\hline
\end{tabular}

Tabla 3. Desvíos estándar de los valores obtenidos para cada pipeta.

R epetibilidad

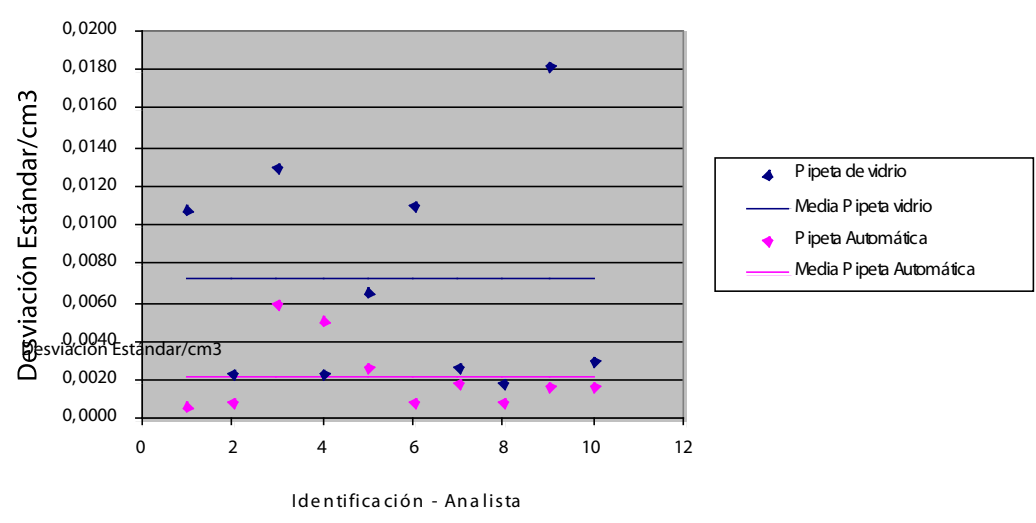

Figura 3. Gráfico de desvíos estándar. 


\section{Discusión}

- En el caso de la pipeta aforada, cuatro de los 10 analistas presentan errores normalizados mayores que 1 . Tres de ellos corresponden a analistas que han ingresado en el último año.

- En el caso de la pipeta automática todos los errores normalizados son inferiores a 1.

- Se infiere que la pipeta automática tiene mejor reproducibilidad.

- Asimismo es posible ver que en el caso de las pipetas automáticas los valores de repetibilidad son mejores en este caso.

A los efectos de analizar los resultados obtenidos y tomar las acciones correctivas necesarias, en caso de resultados no conformes para el uso propuesto es importante tener en cuenta algunas de las posibles causas de errores (sistemáticos en su mayoría).

En el caso de la pipeta aforada:

- Errores en el enrase.

- Diferencias en la forma de vaciado de la pipeta respecto a la especificada en el procedimiento, sobre todo en la descarga final, ya que se debe dejar el tiempo de escurrido especificado y no realizar ningún movimiento brusco que provoque la descarga de las últimas gotas que no caen por gravedad.

Considerados estos errores, en la mayoría de los casos se obtendrían valores superiores a los que se obtienen si se sigue el procedimiento en todos sus términos.

En el caso de la pipeta automática:

- Diferencias en la forma y la fuerza utilizadas para presionar el mecanismo de vaciado de la misma.

En ambas pipetas:

- Errores en las mediciones de temperatura.

- Errores originados por la calidad del agua destilada utilizada.

Se observó que en el caso de la pipeta aforada hubo problemas de manipulación por parte de algunos analistas, que hacen que la reproducibilidad de los resultados no sea buena. Se eligió una pipeta de $1 \mathrm{ml}$ clase $\mathrm{B}$, de formato tal que los posibles errores de manipulación se vieran amplificados, para evidenciarlos y corregirlos. Esta evaluación se realizó en la reunión de discusión de resultados, en la cual se discutieron las posibles causas de los errores constatados, planteándose todas las posibilidades evaluadas. Se detectó que los valores que están por encima se deben en general a que se realizó algún movimiento brusco de la pipeta, el cual provocó la salida de la última porción de agua de su interior, que en otras condiciones no se hubiera dado. Pueden haber existido problemas también en la visualización del menisco. Se resolvió, entonces, proceder a un entrenamiento del personal para unificar los criterios de uso, sobre todo en el caso de bajos volúmenes, ya que en los ensayos de aptitud anteriores, con volúmenes mayores, los resultados han sido buenos. Como en este caso se utilizó una pipeta que amplificaba los problemas, convendría repetir el estudio una vez finalizado el entrenamiento, agregando una pipeta de las mejores de plaza y comparando la performance de ambas con cada analista.

En el caso de la pipeta automática, los valores obtenidos tienen una mayor reproducibilidad, lo que evidencia que el método de descarga es el mismo en todas las ocasiones y no parece depender apreciablemente de la fuerza y la forma en que cada analista realiza la descarga.

Teniendo esto en cuenta y observando los valores de repetibilidad, en la Figura 3 se evidencia que la repetibilidad y reproducibilidad de las mediciones de volumen en el caso de la pipeta automática son mejores que en el caso de la pipeta aforada de vidrio.

\section{Conclusiones}

Cuando se usa material volumétrico aforado, sobre todo cuando sus volúmenes son pequeños, debe procederse a una apropiada selección del mismo.

Debe mantenerse un entrenamiento del personal nuevo en el uso adecuado del material volumétrico y un reentrenamiento en el caso que los resultados lo ameriten. Esta conclusión responde a que tres de los cuatro resultados no conformes obtenidos para la pipeta aforada corresponden a analistas que han ingresado en el último año.

Es recomendable en el caso de querer minimizar las incertidumbres que la persona que usa el material volumétrico sea la misma que lo calibra, debido a que en ambos tipos de pipetas los valores de repetibilidad son mucho mejores que los de reproducibilidad.

El uso de pipetas automáticas disminuye la influencia del analista en los volúmenes entregados, siempre y cuando éstas sean de buena calidad.

\section{Referencias}

- INTERNATIONAL ORGANIZATION FOR STANDARIZATION (Suiza). ISO/IEC 17025: General requirements for the competence of testing and calibration laboratories. Geneva: ISO, 2005.

- INTERNATIONAL ORGANIZATION FOR STANDARIZATION (Suiza). ISO 9001: Quality management systems - requirements. Geneva: ISO, 2000.

- INTERNATIONAL ORGANIZATION FOR STANDARIZATION (Suiza). ISO 4787: Laboratory glassware volumetric glassware methods for use and testing of capacity. Geneva: ISO, 1984.

- INTERNATIONAL ORGANIZATION FOR STANDARIZATION (Suiza). ISO 648: Laboratory glassware one-mark pipettes. Geneva: ISO, 1977.

- INTERNATIONAL ORGANIZATION FOR STANDARIZATION (Suiza). ISO 13528: Statistical methods for use in proficiency testing by interlaboratory comparisons. Geneva: ISO, 2005. [Consulta: 20 de abril de 2008]. Disponible en: http://www.iso.org/iso/isocatalogue/catalogue_tc/catalogue_detail. htm?csnumber $=35664$

- INTERNATIONAL ORGANIZATION FOR STANDARIZATION (Suiza). ISO/IEC Guide 43-1: Proficiency testing by interlaboratory comparisons - Part 1: Development and operation of proficiency testing schemes. Geneva: ISO, 1997. 